The Astrophysical Journal, 215 :800-804, 1977 August 1

(C) 1977. The American Astronomical Society. All rights reserved. Printed in U.S.A.

\title{
GRAPHITE GRAIN SURFACE REACTIONS IN INTERSTELLAR AND PROTOSTELLAR ENVIRONMENTS
}

\author{
MICHAEL J. BARLOW \\ Joint Institute for Laboratory Astrophysics, University of Colorado and National Bureau of Standards \\ AND \\ JOSEPH SILK \\ Institute for Advanced Study \\ Received 1976 December 17
}

\begin{abstract}
Surface reactions on warm $\left(T_{g} \geqslant 60 \mathrm{~K}\right)$ graphite grains between lattice $\mathrm{C}$ atoms and impinging $\mathrm{H}$ and $\mathrm{O}$ atoms, within or in the vicinity of $\mathrm{H}$ II regions, can provide a prolific source of $\mathrm{H}_{2} \mathrm{CO}$ and other interstellar molecules. It is proposed that similar reactions in the primitive solar nebula led to the formation of the organic molecules found in carbonaceous chondrites. From this and related evidence it is argued that most of the solid material in the solar system may have originated as interstellar grains.
\end{abstract}

Subject headings: interstellar: matter - meteors and meteorites

\section{INTRODUCTION}

Graphite grain surface reactions involving chemisorbed atoms can provide a favorable environment for the formation and ejection of complex molecules in interstellar space. The chemisorption of $\mathrm{H}$ atoms on graphite grains with subsequent recombination to $\mathrm{H}_{2}$ has been discussed by Barlow and Silk (1976). Bar-Nun (1975), from the evidence of laboratory experiments, has suggested that the direct reaction of various atoms with $\mathrm{C}$ atoms in graphite grain surfaces could act as an important source of molecules in interstellar space. We here derive some analytic relations governing the reaction of chemisorbed atoms with graphite surfaces and use these relations to discuss the conditions under which such reactions may in fact be of importance in interstellar environments. Nonchemisorbing grains composed of silicate material should not be susceptible to destructive grain surface reactions. It has been suggested that chemical reactions may occur on ice grains (Wickramasinghe and Williams 1968); however, the mechanism proposed has been shown to have an insignificant rate (Barlow 1975, 1977).

\section{BASIC THEORY}

Graphite grains will not react with molecules such as $\mathrm{H}_{2}$ at grain temperatures below $2000 \mathrm{~K}$, at which temperature the sticking probability of $\mathrm{H}_{2}$ is in any case entirely negligible (Beitel 1971, 1972). The principal reaction product of $\mathrm{H}$ atoms with graphite is found to be $\mathrm{CH}_{4}$ (Bar-Nun 1975). The principal reaction products of $\mathrm{O}$ and $\mathrm{N}$ atoms with graphite are $\mathrm{CO}_{2}$ (Bar-Nun 1975; Rosner and Allendorf 1969) and $\mathrm{C}_{2} \mathrm{~N}_{2}$ (Goldstein 1964). The reaction products of atomic $\mathrm{H}$ with graphite are found to evaporate even at low temperatures. Although the reaction products of atomic $\mathrm{O}$ with graphite $\left(\mathrm{CO}\right.$ and $\left.\mathrm{CO}_{2}\right)$ are found to form stable surface oxides (Marsh, O'Hair, and Wynne-Jones 1969), it is expected that in the interstellar case atomic $\mathrm{H}$ will react with the surface $\mathrm{CO}$ complex formed by the initial reaction, with $\mathrm{H}_{2} \mathrm{CO}$ being eventually evaporated from the surface. Similarly, the principal reaction product of atomic $\mathrm{N}$ (with atomic $\mathrm{H}$ ) and graphite should be $\mathrm{HCN}$.

In order to derive a surface reaction rate for a given atomic species with graphite, the surface reaction must be considered in conjunction with the competing mechanism of surface recombination (thermal desorption of atoms chemisorbed on graphite can be neglected since their binding energies are in excess of $1 \mathrm{eV}$; $\mathrm{cf}$. Barlow and Silk 1976). For surface reactions to be significant, the time required for surface recombination of adsorbed atoms must be longer than the time required for reaction with a surface $\mathrm{C}$ atom. We now compute the critical grain temperature for surface reactions to occur.

Let $\Delta E_{m}(i)$ be activation energy for the surface migration of atom $i$ on a graphite surface. The work of Bennett, McCarroll, and Messmer (1971a) predicts $\Delta E_{m}(\mathrm{H})=0.09 \mathrm{eV}$, in good agreement with the experimental determination by King and Wise (1963) of an activation energy of $0.10 \mathrm{eV}$ for $\mathrm{H}_{2}$ recombination on graphite. From Bennett, McCarroll, and Messmer (1971b) $\Delta E_{m}(\mathrm{O})$ is predicted to be equal to $0.70 \mathrm{eV}$, and $\Delta E_{m}(\mathrm{~N})$ is predicted to be equal to $0.89 \mathrm{eV}$. Thus, since the migration activation energies of $\mathrm{O}$ and $\mathrm{N}$ are much larger than that of $\mathrm{H}$, and since $\mathrm{H}$ atoms are generally much more abundant, chemisorbed $\mathrm{O}$ and $\mathrm{N}$ atoms may be considered to be immobile for graphite grain temperatures $T_{g} \leqslant 200 \mathrm{~K}$ and recombination with migrating $\mathrm{H}$ atoms will be the only significant recombination process. In order to estimate the time for recombination with an $\mathrm{H}$ atom, the number of $\mathrm{H}$ atoms on the grain surface at 
any given moment must be known. This number depends on the temperature, since $\mathrm{H}$ atoms are removed by migration and recombination. For low grain temperatures $\left(T_{g} \sim 30 \mathrm{~K}\right)$ quantum mechanical diffusion is probably more important for $\mathrm{H}$ atoms than thermal diffusion (Hollenbach and Salpeter 1970), but the exact form of the quantum mechanical diffusion probability is uncertain at present. Fortunately, it can be shown that as grain temperatures are approached sufficient for surface reaction to occur, thermal diffusion alone suffices to limit the number of adsorbed $\mathrm{H}$ atoms on a grain surface to less than or equal to 2 at any given moment. From Hollenbach and Salpeter (1970), the time $t_{D}$ for a thermally diffusing $\mathrm{H}$ atom to scan a grain surface containing $N$ adsorption sites is given by

$$
t_{D}=N^{2} \nu_{0}^{-1} \exp \left[\Delta E_{m}(\mathrm{H}) / k T\right],
$$

where $\nu_{0}=10^{13} \mathrm{~s}^{-1}$ is the surface vibration frequency of a chemisorbed atom. The mean time $\tau_{s}$ between successive $\mathrm{H}$ atoms sticking to a grain surface is given by

$$
\tau_{s}=\left(\pi a^{2} \bar{V}_{\mathrm{H}} S_{\mathrm{H}} n_{\mathrm{H}}\right)^{-1},
$$

where $a$ is the grain radius and $\bar{V}_{\mathrm{H}}, S_{\mathrm{H}}$, and $n_{\mathrm{H}}$ are the mean thermal velocity, striking probability, and number density of gas-phase $\mathrm{H}$ atoms. By equating $t_{D}$ to $\tau_{s}$ we can obtain the critical grain temperature $T_{g}(2-\mathrm{H})$, above which there are never more than two $\mathrm{H}$ atoms on a grain surface

$$
T_{g}(2-\mathrm{H})=\left[\Delta E_{m}(\mathrm{H}) / k\right] / \ln \left(\nu_{0} \tau_{s} / N^{2}\right) .
$$

Typical values of $T_{g}(2-\mathrm{H})$ are presented in Table 1. For grain temperatures greater than $T_{g}(2-\mathrm{H})$ the time $\tau_{\text {react }}(i)$ for the reaction of an adsorbed atom $i$ with the surface should be compared with the time between successive stickings of $\mathrm{H}$ atoms to the grain, $\tau_{s}$. We have

$$
\tau_{\text {react }}(i)=\nu_{0}^{-1} \exp \left[E_{\text {react }}(i) / k T_{g}\right],
$$

where $E_{\text {react }}(i)$ is the activation energy for surface reaction of an adsorbed atom species $i$. Equating (2) to (4), we obtain $T_{g}$ (react, $i$ ), the critical grain temperature above which reaction with the surface takes place faster than recombination with $\mathrm{H}$ atoms

$$
T_{g}(\text { react, } i)=\left[E_{\text {react }}(i) / k\right] / \ln \left(v_{0} \tau_{s}\right) .
$$

For $\mathrm{H}$ atoms the activation energy for reaction with graphite, $E_{\text {react }}(\mathrm{H})$, is equal to $0.24 \mathrm{eV}$ (Wood and Wise 1962), for $\mathrm{O}$ atoms on graphite $E_{\text {react }}(\mathrm{O})=$ $0.34 \mathrm{eV}$ after correction for surface oxide formation (Marsh, O'Hair, and Wynne-Jones 1969), and for N atoms on graphite $E_{\text {react }}(\mathrm{N})=0.76 \mathrm{eV}$ (Goldstein 1964). Typical values of $T_{g}($ react, $i)$ are displayed in Table 1 for $\mathrm{H}, \mathrm{O}$, and $\mathrm{N}$ atoms on graphite. The table shows that the condition $T_{g}(2-\mathrm{H})<T_{g}$ (react, $i$ ) is always satisfied. Evidently, usually only surface reactions of $\mathrm{H}$ and $\mathrm{O}$ on graphite are of astrophysical interest, since in most situations $T_{g} \leqslant 100 \mathrm{~K}$.

At grain temperatures $T_{g}>T_{g}$ (react, $\mathrm{H}$ ) the molecule formation rate per unit volume due to surface reactions with incident $\mathrm{H}$ atoms can be expressed as

$$
R_{M(\mathrm{C}, \mathrm{H})}=\frac{1}{4} n_{g} \sigma_{g} S_{\mathrm{H}} n_{\mathrm{H}} V_{\mathrm{H}},
$$

where $n_{g}$ is the number density of grains and $\sigma_{g}$ is the cross section of a grain. The factor $\frac{1}{4}$ arises because four $\mathrm{H}$ atoms are needed in order to remove one lattice atom. The sticking probability $S_{\mathrm{H}}$ will be approximately unity at low grain and gas temperatures, but will be much reduced in an $\mathrm{H}$ il region [since the mean energy of an incident photon $(\sim 1.3 \mathrm{eV})$ exceeds the absorption potential $\left.E_{\text {ads }}(\mathrm{H})=1.1 \mathrm{eV}\right]$. For $T_{\text {gas }} \sim 10^{4} \mathrm{~K}, S_{\mathrm{H}}$ is probably within the range $10^{-3} \leqslant$ $S_{\mathrm{H}} \leqslant 10^{-1}$. Because of the reservoir of free electrons in graphite, incoming ions will be neutralized as they approach the grain surface, and react with the surface as $\mathrm{H}$ atoms. Graphite grains only attain a positive charge close to the exciting star of an $\mathrm{H}$ il region (Moorwood and Feuerbacher 1975), so ion collision rates will not be substantially reduced by grain charging. Indeed, Hollenbach (1976, private communication) points out that a small positive potential will actually enhance the sticking probability.

As a rough interpolation, let us set

\begin{tabular}{|c|c|c|c|c|c|c|}
\hline$T_{\text {gas }}(\mathrm{K})$ & $n_{\mathrm{H}}\left(\mathrm{cm}^{-3}\right)$ & $S_{\mathrm{H}}$ & $T_{g}(2-\mathrm{H})^{*}$ & $T_{g}($ react, $\mathrm{H})$ & $T_{g}($ react, $\mathrm{O})$ & $T_{g}($ react, $\mathrm{N})$ \\
\hline $10^{4}$ & 10 & 0.1 & 56 & 67 & 95 & 213 \\
\hline $10^{4}$ & 10 & $10^{-3}$ & 46 & 61 & 86 & 192 \\
\hline $10^{4}$. & $10^{3}$ & 0.1 & 71 & 76 & 114 & 240 \\
\hline $10^{4}$ & $10^{3}$ & $10^{-3}$ & 56 & 67 & 95 & 213 \\
\hline $100 \ldots \ldots \ldots$ & 1 & 1 & 50 & 64 & 90 & 202 \\
\hline
\end{tabular}

$$
\begin{array}{rlr}
S_{\mathrm{H}} & =100 / T_{\text {gas }} & \left(T_{\text {gas }}>100 \mathrm{~K}\right) \\
& =1 \quad\left(T_{\text {gas }}<100 \mathrm{~K}\right) .
\end{array}
$$

The molecule formation rate can now be expressed as

$$
R_{\mathrm{M}(\mathrm{C}, \mathrm{H})} \approx 2 \times 10^{-17} f n_{\mathrm{H}} n \mathrm{~cm}^{-3} \mathrm{~s}^{-1},
$$

TABLE 1

Critical Grain Temperatures (in kelvins) for Surface Reaction of Atoms with Graphite Grains

* For graphite grains of radius $1.5 \times 10^{-6} \mathrm{~cm}$. 
where

$$
\begin{aligned}
f & =\left(\frac{\sigma_{g} n_{g}}{n} / 6 \times 10^{-22} \mathrm{~cm}^{2}\right)\left(\frac{100 \mathrm{~K}}{T_{\text {gas }}}\right)^{1 / 2}, \\
10^{4} \mathrm{~K} \geqslant T_{\text {gas }} \geqslant 100 \mathrm{~K} & \\
=\left(\frac{\sigma_{g} n_{g}}{n} / 6 \times 10^{-22} \mathrm{~cm}^{2}\right)\left(\frac{T_{\text {gas }}}{100 \mathrm{~K}}\right)^{1 / 2}, & T_{\text {gas }} \leqslant 100 \mathrm{~K} .
\end{aligned}
$$

Here $n$ is the number density of hydrogen nuclei and $n_{g} \sigma_{g} / n=6 \times 10^{-22} \mathrm{~cm}^{2}$ is the normal value derived for graphite grains in interstellar space (Barlow and Silk 1976). The quantity $f$ will decrease as the grains are destroyed.

The sticking factor for $\mathrm{O}$ should be larger than for hydrogen atoms at the same temperature. If we assume that the sticking coefficients are equal at the same velocity, and that $O$ is present at its cosmic abundance, we obtain

$$
R_{\mathrm{M}(\mathrm{C}, 0)} \approx 6 \times 10^{-20} \mathrm{fn}^{2} .
$$

Under ordinary interstellar conditions the temperature of graphite is $\leqslant 33 \mathrm{~K}$ (Leung 1975), so surface reactions will not be important. However, in certain specific environments graphite grain surface reactions can occur. We consider three examples of environments where sufficiently hot grains may be present.

\section{APPLICATIONS}

\section{a) Warm Molecular Clouds}

Molecular clouds around $\mathrm{H}$ II regions are often extended infrared sources, typical observed grain and $\mathrm{CO}$ brightness temperatures being $\sim 55-100 \mathrm{~K}$. The best studied example is the Orion molecular cloud, where the heat input to the gas is considered to be due to radiation absorbed by the grains from stars and their associated $\mathrm{H}$ II regions which are embedded in the cloud. Thus $T_{g}$ may exceed $T_{\text {gas }}$. At moderate values of $\tau_{v}$ there will still be substantial amounts of atomic $\mathrm{H}$ and $\mathrm{O}$, yet the photodissociation rates of such molecules as $\mathrm{CH}_{4}$ and $\mathrm{H}_{2} \mathrm{CO}$ are greatly reduced [e.g., at $\tau_{v}=0, \quad \beta \sim(50 \mathrm{yr})^{-1} ;$ at $\tau_{v}=2, \beta \sim$ $\left(10^{4} \mathrm{yr}\right)^{-1}$; and at $\tau_{v}=4, \beta \sim\left(10^{6} \mathrm{yr}\right)^{-1}$ for these molecules (Stief et al. 1972)]. Graphite grains which are sufficiently warm to react with $\mathrm{O}$ and $\mathrm{H}$ atoms would not have ice mantles, because of photodesorption (Barlow 1975, 1977) and evaporation. Surface reactions with warm graphite grains should lead to hydrocarbon abundances of the order of

$$
\frac{\mathrm{CH}_{4}}{\mathrm{H}+2 \mathrm{H}_{2}}=3 \times 10^{-8} f n_{\mathrm{H}} \exp \left(2.5 \tau_{v}\right)
$$

For the case of $\mathrm{H}_{2} \mathrm{CO}$ formation by surface reaction with $\mathrm{O}$ atoms it is found that if $\mathrm{O}$ has a cosmic abundance and $n_{\mathrm{H}} / n \sim 10^{-3}$, then the rate limiting process is reaction of the surface complex with $\mathrm{H}$ atoms. Since two $\mathrm{H}$ atoms are required for every $\mathrm{H}_{2} \mathrm{CO}$ molecule, we obtain

$$
\frac{\mathrm{H}_{2} \mathrm{CO}}{\mathrm{H}+2 \mathrm{H}_{2}}=6 \times 10^{-8} f n_{\mathrm{H}} \exp \left(2.5 \tau_{v}\right) .
$$

The lifetime of a graphite grain of radius $\sim 1.5 \times 10^{-6} \mathrm{~cm}$ under these conditions is $\sim 3 \times 10^{5}$ years, so for molecular cloud ages less than $10^{5}$ years, $f \sim 1$. Since formation of $\mathrm{H}_{2} \mathrm{CO}$ by the mechanism will cease for graphite grain temperatures less than $\sim 90 \mathrm{~K}$ (Table 1 ), an enhanced abundance of $\mathrm{H}_{2} \mathrm{CO}$ should be observed in a fairly thin shell around the $\mathrm{H}$ II region. Assuming that the grains are sufficiently warm if $\tau_{v} \leqslant 1$ and taking $n \sim 10^{3} \mathrm{~cm}^{-3}$ and $n_{\mathrm{H}} / n=10^{-3}$, then

$$
\mathrm{H}_{2} \mathrm{CO} / \mathrm{H}+2 \mathrm{H}_{2} \sim 6 \times 10^{-8}-7 \times 10^{-7}
$$

in the shell.

\section{b) $\mathrm{H}$ II Regions}

Molecules may also be produced by surface reactions in the outer parts of $\mathrm{H}$ II regions. The predicted abundance of $\mathrm{CH}_{4}$, assuming that photodestruction rates are enhanced over those due to the mean interstellar field by a factor $\gamma \sim 20$, is

$$
\frac{\mathrm{CH}_{4}}{\mathrm{H}} \sim 1.5 \times 10^{-9} f n \text {. }
$$

Photodissociation of $\mathrm{CH}_{4}$ will lead to the production of such observable molecules as $\mathrm{CH}$ and $\mathrm{CH}^{+}$. Since $\beta(\mathrm{CH}) \approx \beta\left(\mathrm{CH}_{4}\right)$ (Stief et al. 1972; Black, Dalgarno, and Oppenheimer 1975), we obtain $\mathrm{CH} / \mathrm{H} \approx$ $1.5 \times 10^{-9} \mathrm{fn}$. The dominant destruction mechanism for $\mathrm{CH}^{+}$will be dissociative recombination, $\mathrm{CH}^{+}+e \rightarrow$ $\mathrm{C}+\mathrm{H}$. We adopt a recombination rate $\alpha=2.4 \times$ $10^{-9} \mathrm{~cm}^{3} \mathrm{~s}^{-1}$ for a $10^{4} \mathrm{~K} \mathrm{H}$ II region (the dissociative recombination rate might in fact be higher; see Smith, Liszt, and Lutz 1973). Thus we have $\beta(\mathrm{CH}) / \alpha\left(\mathrm{CH}^{+}\right) n=$ $4 / n$; and since $70 \%$ of $\mathrm{CH}$ photodissociations produce $\mathrm{CH}^{+}$, we obtain $\mathrm{CH}^{+} / \mathrm{H}=4 \times 10^{-9} f$.

For the case of $\zeta \mathrm{Oph}, n \approx 3 \mathrm{~cm}^{-3}$ and $N_{\mathrm{H}}=$ $1.6 \times 10^{20} \mathrm{~cm}^{-2}$ in the circumstellar $\mathrm{H}$ II region (Morton 1975), yielding $N_{\mathrm{CH}} \approx 7 \times 10^{11} f \mathrm{~cm}^{-2}$ and $N_{\mathrm{CH}^{+}} \approx 6 \times 10^{11} \mathrm{f} \mathrm{cm}^{-2}$, with $f \leq 0.1$, whereas Morton finds $N_{\mathrm{CH}}=3 \times 10^{13} \mathrm{~cm}^{-2}$ and $N_{\mathrm{CH}^{+}}=1 \times 10^{13} \mathrm{~cm}^{-2}$. Thus, even if the grains in the circumstellar $\mathrm{H}$ II region of $\zeta \mathrm{Oph}$ were hot enough, the mechanism cannot explain the observed column densities. However, in dense $\mathrm{H}$ il regions detectable amounts of these molecules may be produced by grain surface reactions. Typical lifetimes of graphite grains of radius $1.5 \times$ $10^{-6} \mathrm{~cm}$ in $\mathrm{H}$ II regions will be $5 \times 10^{6} / n$ years.

\section{c) Protostellar Clouds}

Several theories for the origin of solid material in the solar system envisage the primitive solar nebula as pure gas which, beginning at some temperature $\geqslant 2000 \mathrm{~K}$, began to cool, with various solid materials condensing out as the temperature of the cooling gas 
passed through the appropriate condensation temperatures (e.g., Urey 1952; Larimer 1967; Grossman 1972; Barshay and Lewis 1976). Since the model starts with a pure gas, it is implicitly assumed that all preexisting interstellar grains in the solar nebula had been vaporized beforehand.

This model encounters a difficulty in that for a gas with a solar $\mathrm{C}: \mathrm{O}$ abundance ratio $(\sim 0.5)$, graphite would not condense, whereas graphite inclusions are in fact found in carbonaceous chondrites. Thus it has been suggested that these graphite inclusions are a remnant of interstellar dust (Barshay and Lewis 1976). We here propose that all of the solid material in the solar system originated as interstellar dust and that the graphite component of the original interstellar grain mixture was mostly converted into organic molecules (such as are found in carbonaceous chondrites) by means of grain surface reactions. Cameron (1973a) and Hoyle and Wickramasinghe (1976) have postulated that part of the solar solid material, namely the carbonaceous chondrites, originated as interstellar dust, but they proposed formation mechanisms different from that proposed here for the origin of the organic material in these chondrites.

A gravitationally collapsing protostellar cloud, after a period of isothermal collapse, will eventually acquire a centrally peaked temperature distribution (Larson 1969) and a characteristic radius within which temperatures will be high enough for graphite grain surface reactions to occur. Because of the high ambient densities, conversion of the graphite grains into carboncontaining molecules will be rapid once the reactions commence. The initial surface reactions would be expected to produce fairly simple molecules, but these simple molecules would rapidly be converted to more complex forms by means of Fischer-Tropsch catalytic type reactions on the surfaces of neighboring iron, magnetite, and silicaceous grains (Anders, Hayatsu, and Studier 1973). Various "cosmothermometers" indicate formation temperatures for the carbonaceous chondrites of 360-400 K (Anders, Hayatsu, and Studier 1973) and a corresponding density of $n_{\mathrm{H}_{2}} \sim$ $10^{14} \mathrm{~cm}^{-3}$. If we assume that $n_{\mathrm{H}} / n_{\mathrm{H}_{2}} \sim 10^{-6}$ (Iglesias 1976), then the values of $T_{g}$ (react, $\left.i\right)$ for $\mathrm{H}, \mathrm{O}$, and $\mathrm{N}$ are 100,145 , and $330 \mathrm{~K}$, respectively, so reactions involving all three of these species would proceed. ${ }^{1}$ Simultaneously with these reactions, grain clumping and aggregation would be expected to be taking place. Any graphite grains which found themselves in the interior of a clump of silicaceous or metallic grains would be protected against further surface reactions and would survive as inclusions. The carbon-bearing molecules produced by surface reactions would also be expected to accrete onto these grain clumps so that

\footnotetext{
${ }^{1}$ During the process of cloud collapse the initially low temperatures $(\sim 10 \mathrm{~K})$ and high ambient densities would be expected to lead to the formation of icy mantles on grains in the cloud. However, the later rise in gas and grain temperatures would lead to the evaporation of those mantles for temperatures $\geqslant 100-200 \mathrm{~K}$, allowing surface reactions to proceed on the graphite grains.
}

the overall composition would resemble that of the carbonaceous chondrites.

The derived formation temperatures of carbonaceous chondrites are well below the vaporization temperatures of the metallic and silicaceous components. We would argue that the presence of graphite inclusions is evidence that the original interstellar grain mixture was not vaporized in the region of formation of the carbonaceous chondrites, since graphite grains would have been unable to survive if the silicaceous component of the dust had been vaporized. ${ }^{2}$ Thus in our model the various formation temperatures derived for the carbonaceous chondrites would represent the maximum temperatures reached in the localities where they formed. In the scenario of Lewis (1974) and Barshay and Lewis (1976) the formation temperatures of the planets and asteroids represent the temperature to which the solar nebula had locally cooled when these bodies accreted and separated out. Extending the argument for the carbonaceous chondrites, we would suggest instead that these temperatures represent the maximum temperatures reached in the regions where the various bodies formed. Grain materials with condensation temperatures less than a particular formation temperature were vaporized and thus escaped being accreted. Present theoretical models of protostellar evolution are not sufficiently precise to be able to give definitive radial temperature distributions in a protostar nebula; and as discussed by Cameron $(1973 b)$, the present radial distances of solar system bodies may be significantly less than the radial distances at which they formed. Cameron (1975) has also proposed, on the basis of protostellar collapse models, that a large part of the solid material in the solar system originated as interstellar grains.

Our proposal of an interstellar grain origin for the solid material in the solar system is consistent with the proposals by Black (1972), Clayton, Grossman, and Mayeda (1973), and Clayton (1975) that isotopic anomalies discovered in certain meteorite samples are due to inclusions of interstellar grains which condensed in regions of unusual chemical abundance, such as nova or supernova envelopes; a certain small fraction of interstellar grains might have such an origin, and perfect mixing with the rest of the grain mixture would not be expected.

Herbig (1970) has proposed that a significant source of interstellar grains could be provided by the formation of dust in protostellar nebulae followed by the ejection of some fraction into the interstellar medium. We would argue from the evidence above that protostars of solar mass are not a source of new grains unless the ejection efficiency of any newly formed grains is much larger than that of preexisting grains. However, protostellar environments may be important in altering the compositions of grains (e.g., through the oxidation of iron as suggested by Cameron $1973 a$, or the hydration of silicates) and be of importance also

${ }^{2}$ Since most heavy elements in the interstellar medium are already tied up in refractory grain cores (cf. Field 1974; Barlow and Silk 1977), no condensation of new grains would be expected in these regions of the protosolar nebula. 
in modifying the grain size distributions (cf. Burke and Silk 1976, who also proposed that nebulae around protostars of mass greater than about $5 M_{\odot}$ may be a significant source of interstellar grains).
We wish to acknowledge partial support of this research by NSF grant AST 72-05026 A03 (M. J. B.) and NASA grant NGR-05-003-578 (J. S.).

\section{REFERENCES}

Anders, E., Hayatsu, R., and Studier, M. H. 1973, Science, 182, 781 .

Barlow, M. J. 1975, Ph.D. thesis, University of Sussex.

-. 1977, in preparation.

Barlow, M. J., and Silk, J. 1976, Ap. J., 207, 131. 1977, Ap. J. (Letters), 211, L83.

Bar-Nun, A. 1975, Ap. J., 197, 341.

Barshay, S. S., and Lewis, J. S. 1976, Ann. Rev. Astr. Ap., 14, 81.

Beitel, G. A. 1971, J. Vac. Sci. Technol., 8, 647.

. 1972, J. Vac. Sci. Technol., 9, 370.

Bennett, A. J., McCarroll, B., and Messmer, R. P. 1971a, Surface Sci., 24, 191.

. 1971b, Phys. Rev., B3, 1397.

Black, D. C. 1972, Geochim. Cosmochim. Acta, 36, 377.

Black, J. H., Dalgarno, A., and Oppenheimer, M. 1975, Ap. J., 199, 633.

Burke, J. R., and Silk, J. 1976, Ap. J., 210, 341.

Cameron, A. G. W. 1973a, Proc. IAU Symposium No. 52, Interstellar Dust and Related Topics, ed. J. M. Greenberg and H. C. van de Hulst (Dordrecht: Reidel), p. 545.

$1973 b$, Icarus, 18, 407.

1975, in The Dusty Universe, ed. G. B. Field and A. G. W. Cameron (New York: Academic Press), p. 1.

Clayton, D. D. 1975, Ap. J., 199, 765.

Clayton, R. N., Grossman, L., and Mayeda, T. K. 1973, Science, 182, 485 .

Field, G. B. 1974, Ap. J., 187, 453.

Goldstein, H. W. 1964, J. Phys. Chem., 68, 39.

Grossman, L. 1972, Geochim. Cosmochim. Acta, 36, 597.

Herbig, G. H. 1970, Mém. Soc. Roy. Sci. Liège, 19, 13.

Hollenbach, D., and Salpeter, E. E. 1970, J. Chem. Phys., 53, 79.

Hoyle, F., and Wickramasinghe, N. C. 1976, Nature, 264, 45. Iglesias, E. 1976, preprint.

King, A. B., and Wise, H. 1963, J. Phys. Chem., 67, 1163.

Larimer, J. W. 1967, Geochim. Cosmochim. Acta, 31, 1215

Larson, R. B. 1969, M.N.R.A.S., 145, 271.

Leung, C. M. 1975, Ap. J., 199, 340.

Lewis, J. S. 1974, Science, 186, 440.

Marsh, H., O'Hair, T. E., and Wynne-Jones, W. F. K. 1969, Carbon, 7, 555

Moorwood, A. F. M., and Feuerbacher, B. 1975, Ap. Space Sci., 34, 137.

Morton, D. C. $1975, A p . J ., 197,85$

Rosner, D. E., and Allendorf, H. D. 1969, Carbon, 7, 515

Smith, W. H., Liszt, H. S., and Lutz, B. L. 1973, Ap. J., 183, 69.

Stief, L. J., Donn, B., Glicker, S., Gentiau, E. P., and Mentall, J. E. 1972, Ap. J., 171, 21.

Urey, H. C. 1952, Geochim. Cosmochim. Acta, 2, 269.

Wickramasinghe, N. C., and Williams, D. A. 1968, Observatory, 88, 272.

Wood, B. J., and Wise, H. 1962, J. Phys. Chem., 66, 1049.

Michael J. Barlow: Joint Institute for Laboratory Astrophysics, University of Colorado, Boulder, CO 80309 JoSEPH SILK: Astronomy Department, University of California, Berkeley, CA 94720 